\title{
Magnetic Field Structure of Dense Cores Using Spectroscopic Methods
}

\section{Auddy, Sayantan}

2019-02-20

Auddy , S , Myers , P C , Basu , S, Harju , J , Pineda , J E \& Friesen , R K 2019 , ' Magnetic

Field Structure of Dense Cores Using Spectroscopic Methods ' , Astrophysical Journal , vol. 872 , no. 2 , 207 . https://doi.org/10.3847/1538-4357/ab0086

http://hdl.handle.net/10138/303514

https://doi.org/10.3847/1538-4357/ab0086

unspecified

publishedVersion

Downloaded from Helda, University of Helsinki institutional repository.

This is an electronic reprint of the original article.

This reprint may differ from the original in pagination and typographic detail.

Please cite the original version. 


\title{
Magnetic Field Structure of Dense Cores Using Spectroscopic Methods
}

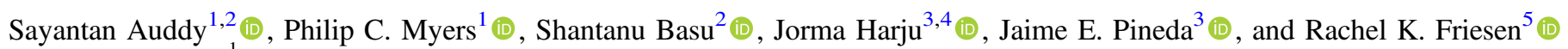 \\ ${ }^{1}$ Harvard-Smithsonian Center for Astrophysics, 60 Garden Street, Cambridge, MA 02138, USA; sauddy3@uwo.ca \\ ${ }^{2}$ Department of Physics and Astronomy, The University of Western Ontario, London, ON N6A 3K7, Canada \\ ${ }^{3}$ Max-Planck-Institute for Extraterrestrial Physics (MPE), Giessenbachstr. 1, D-85748 Garching, Germany \\ ${ }^{4}$ Department of Physics, P.O. Box 64, FI-00014 University of Helsinki, Finland \\ 5 National Radio Astronomy Observatory, Charlottesville, VA 22903, USA \\ Received 2018 September 21; revised 2018 December 22; accepted 2019 January 18; published 2019 February 26
}

\begin{abstract}
We develop a new "core field structure" (CFS) model to predict the magnetic field strength and magnetic field fluctuation profile of dense cores using gas kinematics. We use spatially resolved observations of the nonthermal velocity dispersion from the Green Bank Ammonia survey along with column density maps from SCUBA-2 to estimate the magnetic field strength across seven dense cores located in the L1688 region of Ophiuchus. The CFS model predicts the profile of the relative field fluctuation, which is related to the observable dispersion in the direction of the polarization vectors. Within the context of our model, we find that all of the cores have a transcritical mass-to-flux ratio.
\end{abstract}

Key words: ISM: clouds - ISM: magnetic fields - stars: formation

\section{Introduction}

Stars form in dense cores embedded within interstellar molecular clouds (Lada et al. 1993; Williams et al. 2000; André et al. 2009). Dense cores are well studied observationally from molecular spectral line emission (Myers \& Benson 1983; Benson \& Myers 1989; Jijina et al. 1999), infrared absorption (Teixeira et al. 2005; Lada et al. 2007; Machaieie et al. 2017), and submillimeter dust emission (Ward-Thompson et al. 1994; Kirk et al. 2005; Marsh et al. 2016).

Cores may form in multiple ways including fragmentation of over-dense regions that are typically filaments and sheets (Basu et al. 2009a, 2009b) within turbulent magnetized clouds. Depending on the ambient initial conditions, they can form either as a result of spontaneous gravitational contraction (Jeans 1929; Larson 1985, 2003) or by rapid fragmentation due to preexisting turbulence (Padoan et al. 1997; Klessen 2001; Gammie et al. 2003). Another scenario is the formation of cores in magnetically supported clouds due to quasistatic ambipolar diffusion, i.e., gravitationally induced drift of the neutral species with respect to ions (Mestel \& Spitzer 1956; Mouschovias 1979; Shu et al. 1987). However, a more recent view is that both supersonic turbulence and gravitationally driven ambipolar diffusion are significant in the process of core formation (e.g., Nakamura \& Li 2005; Kudoh \& Basu 2011, 2014; Chen \& Ostriker 2014; Auddy et al. 2018).

Dense cores often have nonthermal contributions to line width that are small compared to the thermal values (Rydbeck et al. 1977; Myers 1983; Caselli et al. 2002). These observations imply a transition from a primarily nonthermal line width in low-density molecular cloud envelopes to a nearly thermal line width within dense cores. This is termed as "a transition to velocity coherence" (Goodman et al. 1998). A sharp transition between the coherent core and the dense turbulent gas surrounding the B5 region in Perseus was found using $\mathrm{NH}_{3}$ observations from the Green Bank Telescope (GBT) by Pineda et al. (2010). It has been suggested that this transition arises from damping and reflection of MHD waves (Pinto et al. 2012).
An important question is whether a transition from magnetic support of low-density regions to gravitational collapse of dense regions is physically related to the transition to coherence. Furthermore, how is the magnetic field strength affecting the nonthermal line width in the low-density region, and is this related to the velocity transition? If so, can one estimate the magnetic field strength and its radial variation across a dense core using such observations?

Accurate measurement of the magnetic field is one of the challenges of observational astrophysics. Several methods exist that probe the magnetic field in the interstellar medium, such as Zeeman detection (e.g., Crutcher 1999), dust polarization (Hoang \& Lazarian 2008), and Faraday rotation (Wolleben \& Reich 2004). While each method has its own limitations (Crutcher 2012), sensitive observations of dust polarization can describe the structure of the plane-of-sky magnetic field and can estimate its strength. According to the dust alignment theory (Andersson et al. 2015), the elongated interstellar dust grains tend to align with their minor axis parallel to the magnetic field. Dust polarization observations from thermal emission or extinction of background starlight provide a unique way to probe the magnetic field morphology in the ISM, including collapsing cores in molecular clouds.

In addition to getting the field morphology, there are various methods to estimate the magnetic field strength. One of the popular techniques is the Davis-Chandrasekhar-Fermi (DCF) method (Davis \& Greenstein 1951; Chandrasekhar \& Fermi 1953) that estimates the field strength using measurements of the field dispersion (about the mean field direction), gas density, and one-dimensional nonthermal velocity dispersion. Dust polarization, however, can be weak in the centers of dense cores where the dust grains are well shielded from the radiative torques necessary to move the grains into alignment with the magnetic field (e.g., see Lazarian \& Hoang 2007).

Recently, Myers et al. (2018) have extended the spherical flux-freezing models of Mestel (1966) and Mestel \& Strittmatter (1967) to spheroidal geometry, allowing quantitative estimates of the magnetic field structure in a variety of spheroidal shapes and orientations. In these models, the magnetic field energy in 


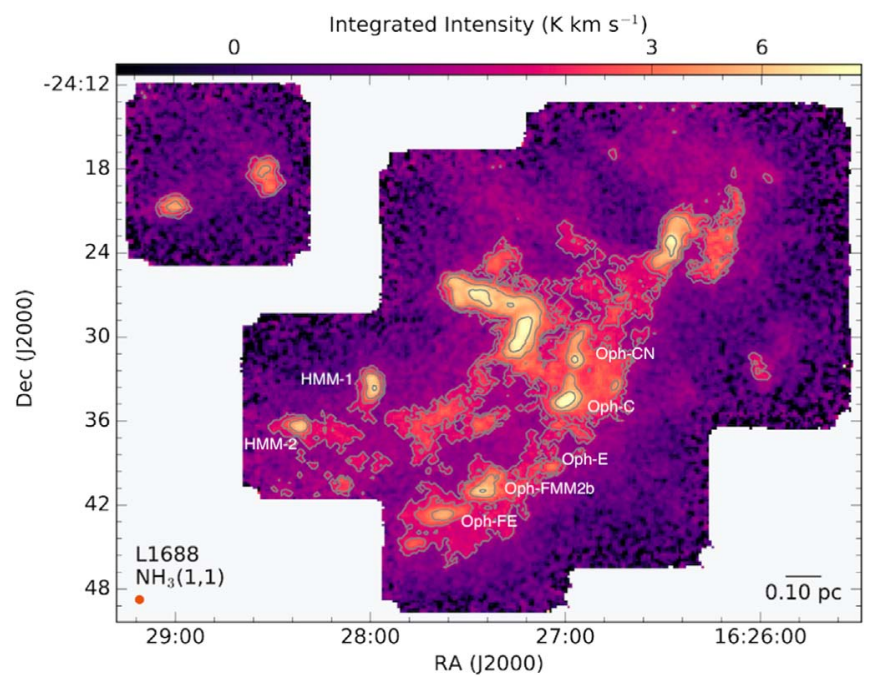

Figure 1. Integrated intensity map of the $\mathrm{NH}_{3}(1,1)$ line for the $\mathrm{L} 1688$ region taken from Friesen et al. (2017). The beam size and scale bar are shown in the bottom left and right corners, respectively. The cores studied in this paper are indicated by name.

the spheroid is weaker than its gravitational energy, allowing gravitational contraction, which drags field lines inward. However, the spheroid magnetic field energy is stronger than its turbulent energy, allowing the field lines to have an ordered "hourglass" structure. These models are useful to test clouds, cores, and filaments that show ordered polarization for the prevalence of flux freezing. They also allow an estimate of the magnetic field structure when the underlying density structure is sufficiently simple and well known.

The present paper is complementary to Myers et al. (2018) since it relies on many of the same assumptions of flux freezing in a centrally condensed star-forming structure. However, it is more specific to dense cores having subsonic line widths. It also relies on additional information, i.e., maps of nonthermal line widths, and on the additional assumption that the nonthermal line widths are due to Alfvénic fluctuations in the magnetic field lines, as in the original studies of DCF.

In this paper, we predict magnetic field structure by analyzing new $\mathrm{NH}_{3}$ observations of multiple cores in the L1688 region in the Ophiuchus molecular cloud. Most of the cores show a sharp transition to coherence with a nearly subsonic nonthermal velocity dispersion in the inner region. We propose a new "Core Field Structure" (CFS) model of estimating the amplitude of magnetic field fluctuations. It incorporates detailed maps from the Green Bank Ammonia Survey (GAS) of the nonthermal line width profiles across a core. The paper is organized in the following manner. The observations of the gas kinematics and the column density are reported in Section 2. In Section 3, we introduce the CFS model and the inferred magnetic field profile. In Section 4, we discuss the limitations of the model. We highlight some of the important conclusions in Section 5.

\section{Observations}

The first data release paper from the GBT survey (Friesen et al. 2017) included detailed $\mathrm{NH}_{3}$ maps of the gas kinematics (velocity dispersion, $\sigma_{v}$ and gas kinetic temperature, $T_{K}$ ) of four regions in the Gould Belt: B18 in Taurus, NGC 1333 in Perseus, L1688 in Ophiuchus, and Orion A North in Orion. The

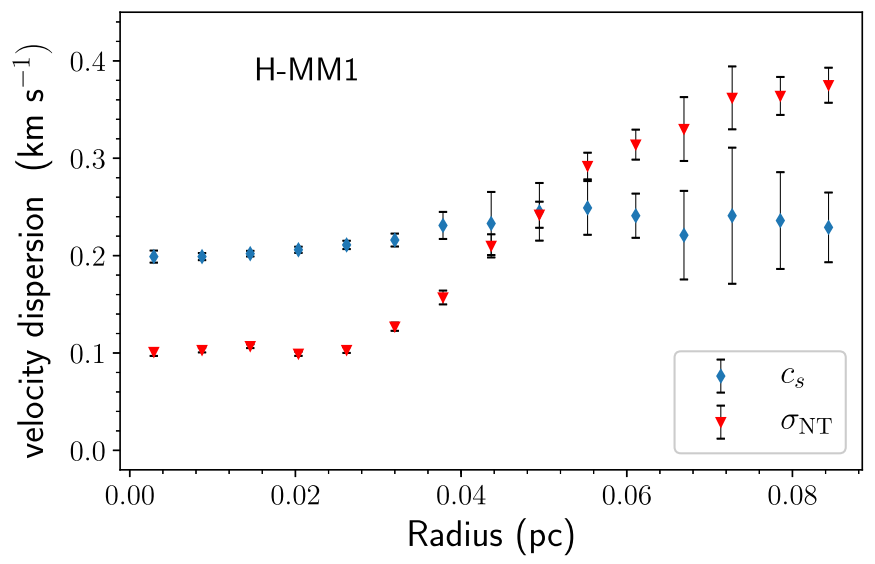

Figure 2. Velocity dispersion profiles of the H-MM1 core calculated using the $\mathrm{NH}_{3}(1,1)$ and $\mathrm{NH}_{3}(2,2)$ spectral line cubes from the GAS survey of the L1688 region of Ophiuchus (Friesen et al. 2017). Here, $c_{\mathrm{s}}$ and $\sigma_{\mathrm{NT}}$ are calculated from annularly averaged spectra, by first aligning the spectra in velocity with the help of the $v_{\mathrm{LSR}}$ map. The transonic radius (where $c_{\mathrm{s}}=\sigma_{\mathrm{NT}}$ ) of H-MM1 can be identified as $r_{0} \simeq 86^{\prime \prime}(0.05 \mathrm{pc}$ at the $120 \mathrm{pc}$ distance of Ophiuchus $)$.

emission from the $\mathrm{NH}_{3}(J, K)=(1,1)$ and $(2,2)$ inversion lines in the $\mathrm{L} 1688$ region of the Ophiuchus cores were obtained using the $100 \mathrm{~m}$ GBT. The observations were done using inband frequency switching with a frequency throw of $4.11 \mathrm{MHz}$, using the GBT $K$-band (upper) receiver and the GBT spectrometer at the front and back end, respectively. L1688 is located centrally in the Ophiuchus molecular cloud. It is a concentrated dense hub (with numerous dense gas cores) spanning approximately $1-2 \mathrm{pc}$ in radius with a mass of $2 \times 10^{3} M_{\odot}$ (Loren 1989). L1688 has over 300 young stellar objects (Wilking et al. 2008) and contains regions of high visual extinction, with $A_{V} \sim 50-100$ mag (e.g., Wilking \& Lada 1983). The mean gas number density of L1688 is approximately a few $\times 10^{3} \mathrm{~cm}^{-3}$.

Submillimeter continuum emission from dust shows that the star formation efficiency of the dense gas cores is $\approx 14 \%$ (Jørgensen et al. 2008). Figure 1 (modified from Figure 6 in Friesen et al. 2017) shows the integrated intensity map of the $\mathrm{NH}_{3}(1,1)$ line for the L1688 region in Ophiuchus, along with the marked cores that are studied in this paper. The map includes four prominent isolated starless cores (including H-MM1 and H-MM2) lying on the outskirts of the cloud, plus more than a dozen local line width minima in the main cloud (mainly in the south-eastern part in regions called Oph-C, E, and F). Many of these minima correspond to roundish starless cores that can be identified on the SCUBA-2 850 micron dust continuum map. The cores indicated as Oph-C, Oph-CN, Oph$\mathrm{E}$, and Oph-FE correspond to source names C-MM3, C-MM11, E-MM2d, and F-MM11, respectively, as mentioned in Pattle et al. (2015). Furthermore, Oph-C, Oph-E, and HMM-1 are classified as starless, while the other cores are known to have a protostar (see Table 1 in Pattle et al. 2015 for more core properties).

\subsection{Velocity Dispersion}

The radial distributions of the velocity dispersions and the kinetic temperatures were calculated from aligned and averaged $\mathrm{NH}_{3}(1,1)$ and $\mathrm{NH}_{3}(2,2)$ spectra. The averages were calculated in concentric annuli, weighting the spectra by the inverse of the rms noise (for example see Figure 12, which demonstrates 

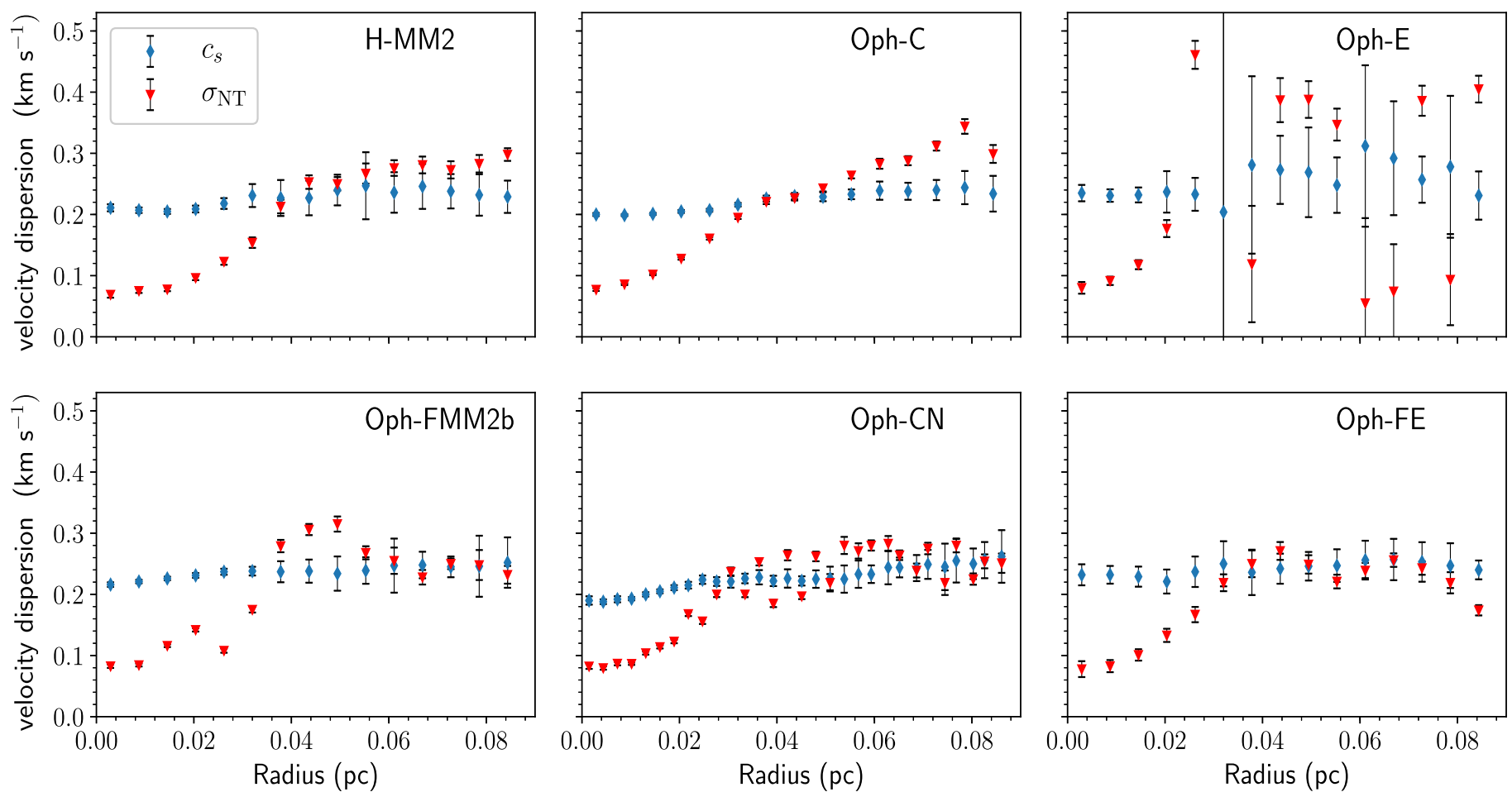

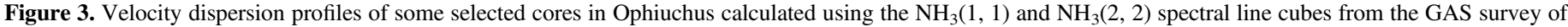

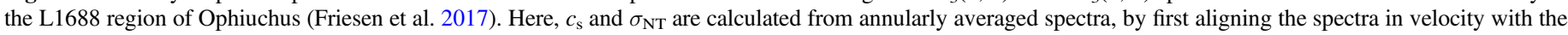
help of the $v_{\mathrm{LSR}}$ map.

annular averaging of spectra for the H-MM1 core). Before the averaging, the spectra were aligned in velocity using LSR velocity maps produced by the reduction and analysis pipeline for the Greenbank $K$-band Focal Plane Array Receiver (Masters et al. 2011; Friesen et al. 2017). ${ }^{6}$

The stacked $\mathrm{NH}_{3}(1,1)$ and $(2,2)$ spectra were analyzed using the standard method described by Ho \& Townes (1983) and, recently, by Friesen et al. (2017). In this method, the velocity, line width, the total optical depth, and the excitation temperature of the $(1,1)$ inversion line are determined simultaneously by fitting a Gaussian function to the 18 hyperfine components. The assumption is that individual hyperfine components have equal excitation temperatures, $T_{\mathrm{ex}}$, beam-filling factors, and line widths.

The column density of molecules in the $(J=1, K=1)$ level depends on $T_{\mathrm{ex}}$ and is proportional to the product of the line width and the total optical depth. The $(2,2)$ inversion line is usually optically thin, and the column density of the molecules in the $(J=2, K=2)$ level is estimated using the integrated intensity of the $(2,2)$ inversion line. It is assumed that the $(2,2)$ inversion line has the same dependence on $T_{\mathrm{ex}}$ and has the same relation to the line width as the $(1,1)$ line. The ratio of the column densities of the $J, K=(1,1)$ and $(2,2)$ levels defines the rotation temperature, $T_{\text {rot }}$. The kinetic temperature $T_{\text {kin }}$ was estimated using the three-level approximation, including levels $J, K=(1,1),(2,2)$, and $(2,1)$, as described by Walmsley \& Ungerechts (1983) and Danby et al. (1988).

The nonthermal velocity dispersion in an averaged spectral line was calculated by subtracting in quadrature the thermal velocity dispersion of ammonia molecules from the total

\footnotetext{
6 The data are available through https://dataverse.harvard.edu/dataverse/ GAS_DR1.
}

velocity dispersion. The errors of the thermal and nonthermal velocity dispersions were calculated by propagating the uncertainties of the variables derived from the averaged spectra. Here, it is assumed that the error in $T_{\text {kin }}$ does not correlate with that of the line width. The dominant uncertainties in the $T_{\text {kin }}$ estimate are related to the optical thickness of the $(1,1)$ line (depending on the relative intensities of the hyperfine components) and the integrated intensity of the $(2,2)$ line. The relative error of the line width is usually very small (a few percent) and has a minor effect on the uncertainty in $T_{\text {kin }}$.

Figure 2 shows the radially averaged isothermal sound speed $c_{\mathrm{S}}$ and nonthermal velocity dispersion $\sigma_{\mathrm{NT}}$ in HMM-1. Here, $c_{\mathrm{s}}=\sqrt{k T / \mu m_{\mathrm{H}}}$ where $T$ is the kinetic temperature, $m_{\mathrm{H}}$ is the mass of a hydrogen atom, and $\mu=2.33$ is the mean molecular mass. Furthermore, $\sigma_{\mathrm{NT}}=\Delta v_{\mathrm{NT}} / \sqrt{2 \ln 2}$, and $\Delta v_{\mathrm{NT}}$ is the nonthermal contribution to the $\mathrm{NH}_{3}$ line width. There is a clear transition point at radius $\approx 86^{\prime \prime}$, where $c_{\mathrm{s}}=\sigma_{\mathrm{NT}}$. We identify this radius as the transonic radius, $r_{\mathrm{c}}$, and consider it to be the core boundary. The nonthermal velocity dispersion is $\approx 0.5 c_{\mathrm{s}}$ inside the core, and it increases steeply to $\approx 2 c_{\mathrm{s}}$ across the core boundary. We use the same prescription to map the thermal and nonthermal velocity dispersion of six other selected cores in L1688. Figure 3 shows the annularly averaged thermal and nonthermal velocity dispersions of all the other selected cores in L1688. We have selected only those cores that have a distinct delineation between thermal/nonthermal line widths $\left(c_{\mathrm{s}}=\sigma_{\mathrm{NT}}\right)$ at a transonic radius $r_{\mathrm{c}}$ with the nonthermal dispersion becoming subthermal toward the center of the core. Outside the transonic radius for some cores (for example Oph-CN and Oph-Fe), the nonthermal dispersion is comparable to the sound speed.

In Table 1, we give the measure of the transonic radius $r_{\mathrm{c}}$ and the corresponding velocity dispersion $\left(\sigma_{\mathrm{NT}}\right)_{\mathrm{c}}$ at $r_{\mathrm{c}}$. The values of $r_{\mathrm{c}}$ and $\left(\sigma_{\mathrm{NT}}\right)_{\mathrm{c}}$ are obtained by interpolating the 


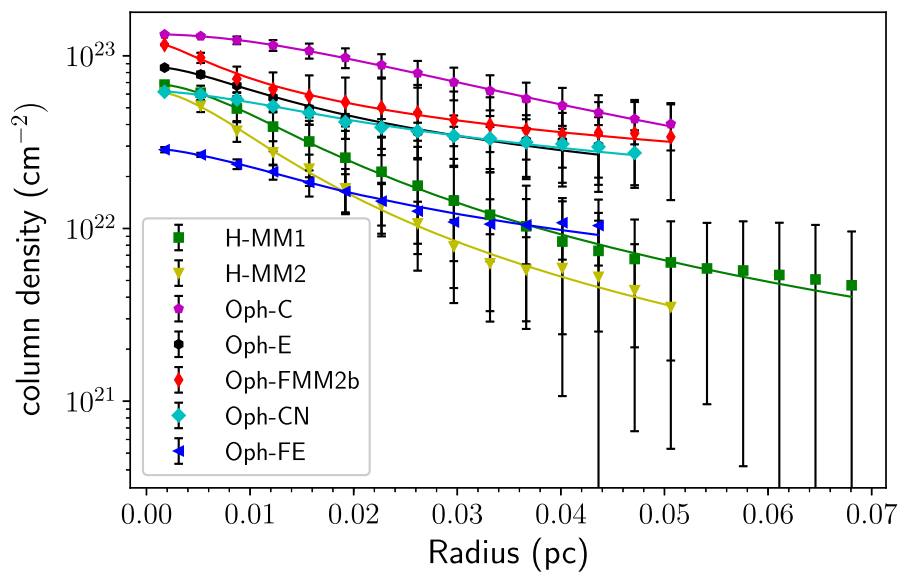

(a)

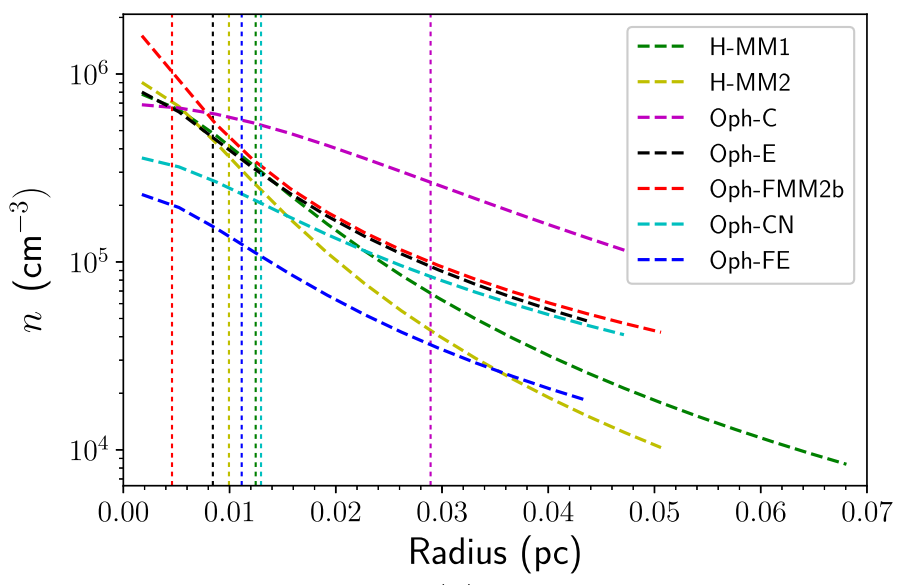

(b)

Figure 4. Left: submillimeter intensities as functions of radial distance from the center of cores in Ophiuchus. The colored markers with error bars indicate averages over concentric annuli and their standard deviations. These are obtained from SCUBA-2 maps at $850 \mu \mathrm{m}$ published by Pattle et al. (2015). The solid curves are fits to the data using the Plummer model. Right: the density profiles as functions of radial distance from the center of cores in L1688. Here, we plot $n(r)$ using the fit parameters (see Table 1). The vertical dotted lines mark the extent of the central flat region $r_{0}$.

Table 1

Core Properties Derived from the Submillimeter Continuum Observations and $\mathrm{NH}_{3}$ Lines Observations

\begin{tabular}{|c|c|c|c|c|c|c|c|c|}
\hline $\begin{array}{l}\text { Core } \\
\text { Name }\end{array}$ & $\begin{array}{l}\text { R.A. 16 } \\
\text { (J2000) }\end{array}$ & $\begin{array}{l}\text { Decl. }-24^{\mathrm{h}} \\
\quad(\mathrm{J} 2000)\end{array}$ & $\begin{array}{c}r_{\mathrm{c}} \\
\left(10^{-2} \mathrm{pc}\right)\end{array}$ & $\begin{array}{c}\left(\sigma_{\mathrm{NT}}\right)_{\mathrm{c}} \\
\left(\mathrm{km} \mathrm{s}^{-1}\right)\end{array}$ & $\begin{array}{c}n_{0} \\
\left(10^{5} \mathrm{~cm}^{-3}\right)\end{array}$ & $\begin{array}{c}r_{0} \\
\left(10^{-2} \mathrm{pc}\right)\end{array}$ & $p / 2$ & $\begin{array}{l}\text { Mass } \\
\left(M_{\odot}\right)\end{array}$ \\
\hline H-MM1 & $27: 58.56$ & $33: 39$ & 5.0 & 0.25 & $8.0 \pm 3.0$ & $1.0 \pm 0.4$ & $1.3 \pm 0.2$ & $\overline{1.7 \pm 0.8}$ \\
\hline H-MM2 & $27: 28.21$ & $36: 27$ & 3.9 & 0.23 & $9.0 \pm 2.0$ & $1.0 \pm 0.2$ & $1.4 \pm 0.2$ & $0.9 \pm 0.3$ \\
\hline Oph-E & $27: 05.80$ & $39: 19$ & 2.2 & 0.24 & $8.0 \pm 3.0$ & $0.8 \pm 0.3$ & $0.9 \pm 0.1$ & $0.6 \pm 0.2$ \\
\hline Oph-FMM2b & $27: 25.10$ & 41:00 & 3.5 & 0.24 & $18.0 \pm 8.0$ & $0.5 \pm 0.2$ & $0.8 \pm 0.1$ & $1.5 \pm 0.7$ \\
\hline Oph-CN & $26: 57.10$ & $31: 47$ & 2.9 & 0.22 & $4.0 \pm 1.0$ & $1.0 \pm 0.5$ & $0.8 \pm 0.1$ & $0.7 \pm 0.2$ \\
\hline
\end{tabular}

Note. The Plummer fit parameters are $n_{0}, r_{0}$, and $p / 2, r_{\mathrm{c}}$ is the transonic radius, and $\left(\sigma_{\mathrm{NT}}\right)_{\mathrm{c}}$ is the velocity dispersion at $r_{\mathrm{c}}$. The final column gives an estimate of the mass of each core.

thermal and nonthermal data points and finding their intersection.

\subsection{Column Density and Density Model}

Figure 4 shows the circularly averaged $850 \mu \mathrm{m}$ intensity profiles of seven cores in L1688 derived from SCUBA-2 maps (see Figure 1 in Pattle et al. 2015). In order to characterize each observed column density profile, we adopt an idealized Plummer model of a spherical core (Arzoumanian et al. 2011) with radial density

$$
\rho(r)=\frac{\rho_{0}}{\left[1+\left(r / r_{0}\right)^{2}\right]^{p / 2}},
$$

where the parameter $r_{0}$ is the characteristic radius of the flat inner region of the density profile, $\rho_{0}=\mu m_{\mathrm{H}} n_{0}$ is the density at the center of the core and $p$ is the power-law index. The column density profile for such a sphere of radius $r$ can be modeled as

$$
\Sigma_{p}(r)=A_{p} \frac{\rho_{0} r_{0}}{\left[1+\left(r / r_{0}\right)^{2}\right]^{\frac{p-1}{2}}},
$$

where $\Sigma=\mu m_{\mathrm{H}} N_{\mathrm{H}_{2}}$ is the observed column density, $N_{\mathrm{H}_{2}}$ is the number column density, and

$$
A_{p}=\int_{-\infty}^{\infty} \frac{d u}{\left(1+u^{2}\right)^{p / 2}}
$$

is a constant. We fit the model profile to the SCUBA-2 $850 \mu \mathrm{m}$ data after they are averaged over concentric circular annuli. For fitting the model to the observational data, $r_{0}, n_{0}$ (number density at the center), and $p$ are treated as free parameters. The left panel in Figure 4 is the Plummer fit to the averaged submillimeter intensities of the concentric annuli of selected cores (with clearest delineation between thermal/nonthermal motions) in L1688 region in Ophiuchus. The results from the fit are summarized in Table 1. On the right panel of Figure 4, we plot the density profile of all the cores (using Equation (1)). For most of the cores, there is a noticeable central flat region of nearly constant density and then a gradual power-law decrease radially outward. The index $p / 2$ is different for each model and varies in the range $0.78<p / 2 \leqslant 1.38$. An estimate of the mass of each core is also given in Table 1. The mass is calculated by integrating the spherical density profile up to the core radius (transonic radius). We run several iterations where the density fit parameters are drawn randomly from respective normal Gaussian distributions with a standard deviation equal to the error range of each parameter. Additionally, for each core, we assume a spread of $10 \%$ for the transonic radius $r_{\mathrm{c}}$ to incorporate the uncertainty (which on average is $\approx 10 \%$ ) in the thermal and nonthermal line widths. The obtained mass distribution is skewed. The process is repeated 100 times and 


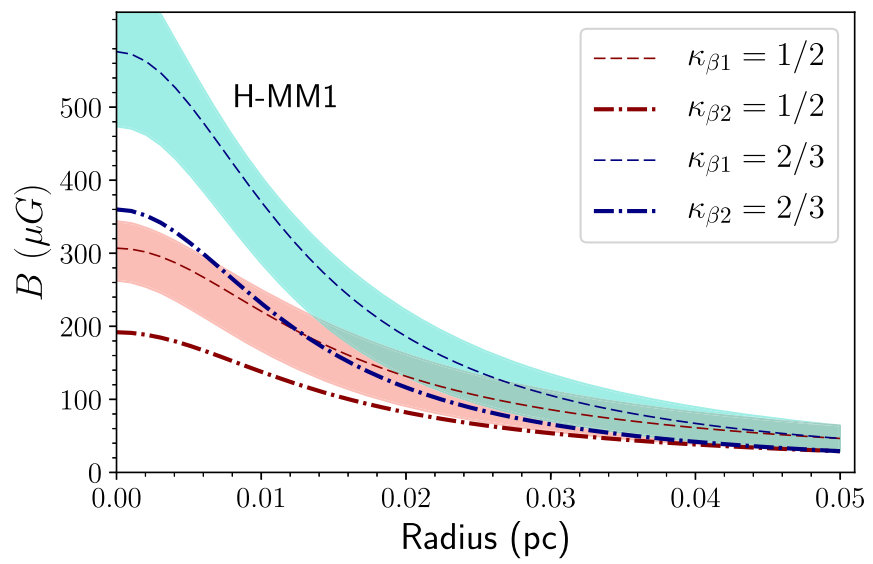

Figure 5. The magnetic field profile of H-MM1 obtained from the CFS model using the observed line widths and density. The red and the blue dashed lines are the magnetic field $B$ for $\kappa=1 / 2$ and $\kappa=2 / 3$, respectively, for the choice of $\beta_{1}=0.5$. The shaded region encloses the first and the third quartile of the distribution obtained using a Monte Carlo analysis. The dotted-dashed red and blue lines are the magnetic field $B$ for $\kappa=1 / 2$ and $\kappa=2 / 3$, respectively, for $\beta_{2}=0.8$.

the uncertainty is calculated from the mean of $S / \sqrt{(2 \ln 2)}$, where $S$ is the semi-interquartile range, for each distribution.

\section{Model}

The CFS model assumes that magnetic field lines are effectively frozen-in to the gas, i.e., the contraction time is shorter then the timescale associated with flux loss (Fiedler \& Mouschovias 1993). The field lines are pinched toward the center of the core due to gravitational contraction. Furthermore, Alfvénic fluctuations are assumed to dominate the nonthermal component of the velocity dispersion. In this section, we discuss the details of the theory and provide justifications. We apply it to the seven selected cores to predict their magnetic field strength profile, the mean magnetic field fluctuation $\delta B$, and the mass-to-flux ratio profile.

\subsection{Core Field Structure}

Our first assumption is that the field strength follows a power-law approximation due to flux freezing. The magnetic field $B(r)$ within the core radius $r_{\mathrm{c}}$ can be written in terms of the observed values as

$$
B(r) / B\left(r_{\mathrm{c}}\right)=\left[\rho(r) / \rho\left(r_{\mathrm{c}}\right)\right]^{\kappa},
$$

where $1 / 2 \leqslant \kappa \leqslant 2 / 3$ (Crutcher 2012) is a power-law index. Here, $B\left(r_{\mathrm{c}}\right)$ is the field strength at the transonic radius. The gas density approaches a near uniform value outside $r_{\mathrm{c}}$. Thus, we do not extend the power-law approximation beyond the core radius. We assume that the core is truncated by an external medium as for a Bonnor-Ebert sphere. Equation (4) approximates various relations obtained from theoretical and numerical models of magnetic cores. Mestel (1966) showed that $\kappa=2 / 3$ in the limit of weak magnetic field and spherical isotropic contraction (which can occur if thermal support nearly balances gravity). Theoretically, $B \propto \rho^{2 / 3}$ relates the mean field and the mean density within a given radius. In Equation (4), we generalize that idea with the approximation that $B \propto \rho^{2 / 3}$ can be applied to obtain the local magnetic field $B(r)$ using the local density $\rho(r)$. This approximation has an associated uncertainty of a factor $\lesssim 2$ as discussed in Section 4.

In the limit of gravitational contraction mediated by a strong magnetic field, Mouschovias (1976a) showed that $\kappa$ is closer to $1 / 2$. In the limit of very strong magnetic field (subcritical massto-flux ratio) models of the ambient molecular cloud (Fiedler \& Mouschovias 1993), ambipolar diffusion leads to the formation of supercritical cores within which $\kappa=1 / 2$. We are only applying Equation (4) within the transonic radius, within which local self-gravity is presumed to be dominant.

To model the nonthermal motions, we assume Alfvénic fluctuations. This means that we ignore possible additional sources of the nonthermal line width, for example unresolved infall motions. The Alfvénic fluctuations obey

$$
\frac{\sigma_{\mathrm{NT}}}{v_{\mathrm{A}}}=\frac{\delta B}{B},
$$

where the Alfvén speed is defined by $v_{\mathrm{A}} \equiv B /(\sqrt{4 \pi \rho})$. This directly leads to

$$
\delta B=\sigma_{\mathrm{NT}} \sqrt{4 \pi \rho} .
$$

We also use Equation (5) to get

$$
B\left(r_{\mathrm{c}}\right)=\frac{\left(\sigma_{\mathrm{NT}}\right)_{\mathrm{c}}}{\beta} \sqrt{4 \pi \rho\left(r_{\mathrm{c}}\right)}
$$

for use in Equation (4) by estimating a value of relative field fluctuation $\beta \equiv \delta B / B$ at $r=r_{\mathrm{c}}$.

Kudoh \& Basu (2003) showed in a simulation with turbulent driving that $\beta$ is restricted to $\lesssim 1$ as highly nonlinear Alfvénic waves quickly steepen and drain energy to shocks and acoustic motions, and that their model cloud evolved to a state in which $\sigma_{\mathrm{NT}} \approx 0.5 v_{\mathrm{A}}$. They found that for a range of different amplitudes of turbulent driving, the value of $\beta$ saturates at a maximum value in the range of $0.5-0.8$. Based on these results, we pick a range $0.5 \leqslant \beta \leqslant 0.8$ at the inner boundary $\left(r=r_{\mathrm{c}}\right)$ of the turbulent region. For simplicity, we demonstrate only the two limiting values $\beta_{1}=0.5$ and $\beta_{2}=0.8$ in Section 3.2.

The nonthermal velocity dispersion arises from transverse Alfvénic waves. However, the observed small variation in $\sigma_{\mathrm{NT}}$ from core to core suggests that $\sigma_{\mathrm{NT}}$ is robust against the likely variation in mean field angle. This is possible because Alfvénic motions are nonlinear in the outer parts of the core. Thus, the Alfvén waves will have magnetic pressure gradients (in $\delta B$ ) that will drive motions along the mean field direction as well. The composite nonthermal line width, accounting for motions in all directions, is expected to be comparable to the mean Alfvén speed within a factor of order 2 (see Figure 13 of Kudoh $\&$ Basu 2003). Thus, the effects of differing viewing angles are relatively small. The CFS model essentially predicts the magnetic field profiles (using Equation (4)) of the dense cores in Ophiuchus. Furthermore, it yields the variation of $\delta B / B$ and the normalized mass-flux ratio within each core profile. We discuss some of the predicted core properties in the next subsection.

\subsection{Core Properties}

Figure 5 shows the magnetic field profile of H-MM1 obtained using the CFS model. The magnetic field $B$ increases radially inward and the ascent is steeper for $\kappa=2 / 3$. For 

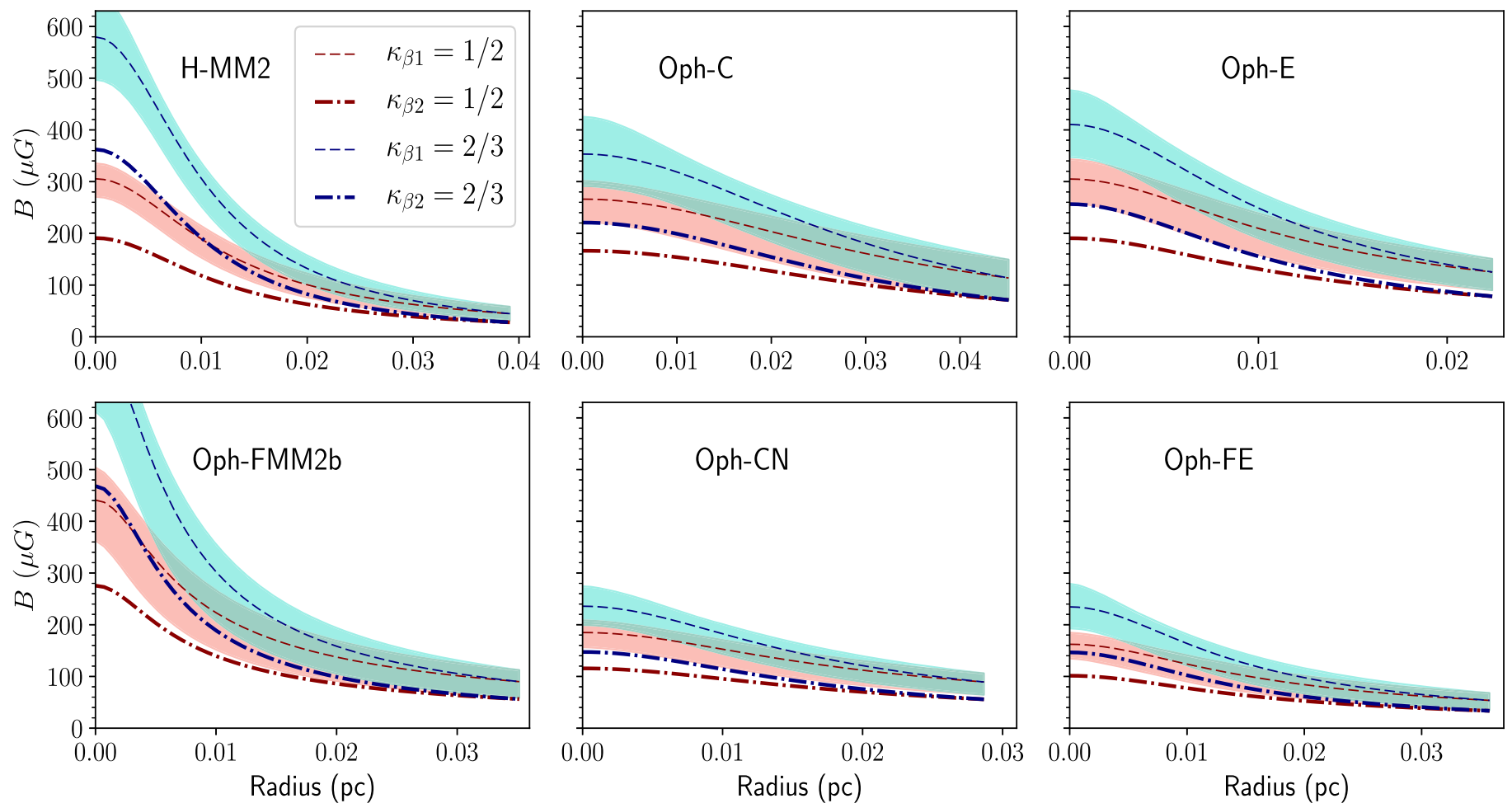

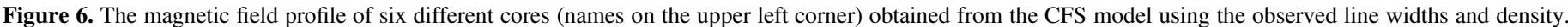

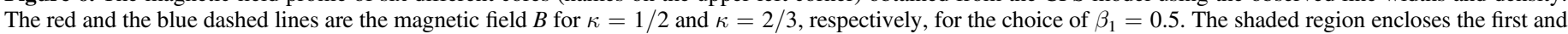

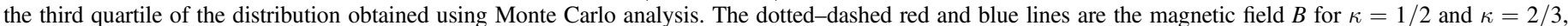
respectively, for $\beta_{2}=0.8$. The magnetic field increases radially inward and the ascent is steeper for $\kappa=2 / 3$.

example, the $B$ value at $r=0.01 \mathrm{pc}$ for $\kappa=2 / 3$ is $\simeq 68 \%$ greater than that for $\kappa=1 / 2$. Similarly, we predict the magnetic field strength profile of all the other cores using the power-law model. Figure 6 shows the field profile as a function of radial distance from the center. Similar to H-MM1, the field strength at a radius of $0.01 \mathrm{pc}$ from the center is greater for $\kappa=2 / 3$ compared to $\kappa=1 / 2$, with a maximum increase of $61 \%$ in H-MM2 and a minimum increase of $19 \%$ in Oph-E. Furthermore, the general increase of the field strength toward the core center can be associated with the pinching of the field lines due to flux freezing. The power-law relation $B \propto \rho^{\kappa}$ for $\kappa=1 / 2$ or $2 / 3$ captures different geometries. For example, $\kappa=2 / 3$ is consistent with a spherical core and $\kappa=1 / 2$ corresponds to flattening along the magnetic field lines.

We use a Monte Carlo analysis, where we run several iterations to evaluate the magnetic field strength using Equation (4). The parameters (for example, $r_{0}, \rho_{0}$, and $p / 2$ ) are randomly picked from a Gaussian distribution with standard deviation equal to the error range of each parameter (see Table 1). Additionally, we assume a variation of $10 \%$ for the values of $\left(\sigma_{\mathrm{NT}}\right)_{\mathrm{c}}$ and $r_{\mathrm{c}}$ to incorporate the uncertainty (on average $\approx 10 \%$ ) in the thermal and nonthermal line widths. The shaded region in both the plots encloses the first and the third quartile of the distribution of magnetic field strength. The dotted curve is the actual model value for $\beta_{1}=0.5$. We repeat a similar analysis for the six other cores in Ophiuchus. There is a significant decrease in the field strength of $\approx 38 \%$ (as indicated by the dotted-dashed lines) for a larger assumed value of field fluctuation (i.e., $\beta_{2}=0.8$ ) at the transonic radius $r_{\mathrm{c}}$. Although there is a systematic dependence of the field strength on the choice of $\beta$, the overall shape of the magnetic field profile remains the same.

Figure 7 shows the fluctuations of the mean magnetic field $\delta B$ and $\delta B / B$ mapped across the H-MM1 core. These are obtained using Equation (6) and the observed nonthermal velocity dispersion data, density, and the modeled magnetic field. The inferred variation of $\delta B / B$ shows a trend very similar to the nonthermal velocity fluctuations. It increases outward as it approaches the transonic radius. Inside the core, $\delta B / B$ decreases to a relatively constant value of $\approx 0.1$. The $\delta B / B$ profile essentially captures the Alfvénic fluctuations across H-MM1. The values of $\delta B / B$ will only correspond to an observed $\delta \theta$ in polarization direction if the observed magnetic field is oriented along the plane of the sky. Figure 8 shows $\delta B$ and $\delta B / B$ for the other cores in Ophiuchus. They all exhibit a very similar trend as H-MM1.

\subsection{The Mass-to-flux Ratio}

In this section, we estimate the normalized mass-to-flux ratio $\mu \equiv M / \Phi /(M / \Phi)_{\text {crit }}$, where $(M / \Phi)_{\text {crit }}=(2 \pi \sqrt{G})^{-1}$ (Nakano \& Nakamura 1978), of the seven cores studied in this paper, assuming a spherically symmetric density profile. The relative strength of gravity and the magnetic field is measured by the mass-to-flux ratio $M / \Phi$. For $M / \Phi>(M / \Phi)_{\text {crit }}$, the cloud is supercritical and can collapse if there is sufficient external pressure. However, for $M / \Phi<(M / \Phi)_{\text {crit }}$ the cloud is subcritical and the field can prevent its collapse as long as magnetic flux freezing applies. An analytic expression for $M / \Phi$ is possible if we assume that the magnetic field lines are threading a spherical core in the plane of the sky. See Appendix B for the derivation. 

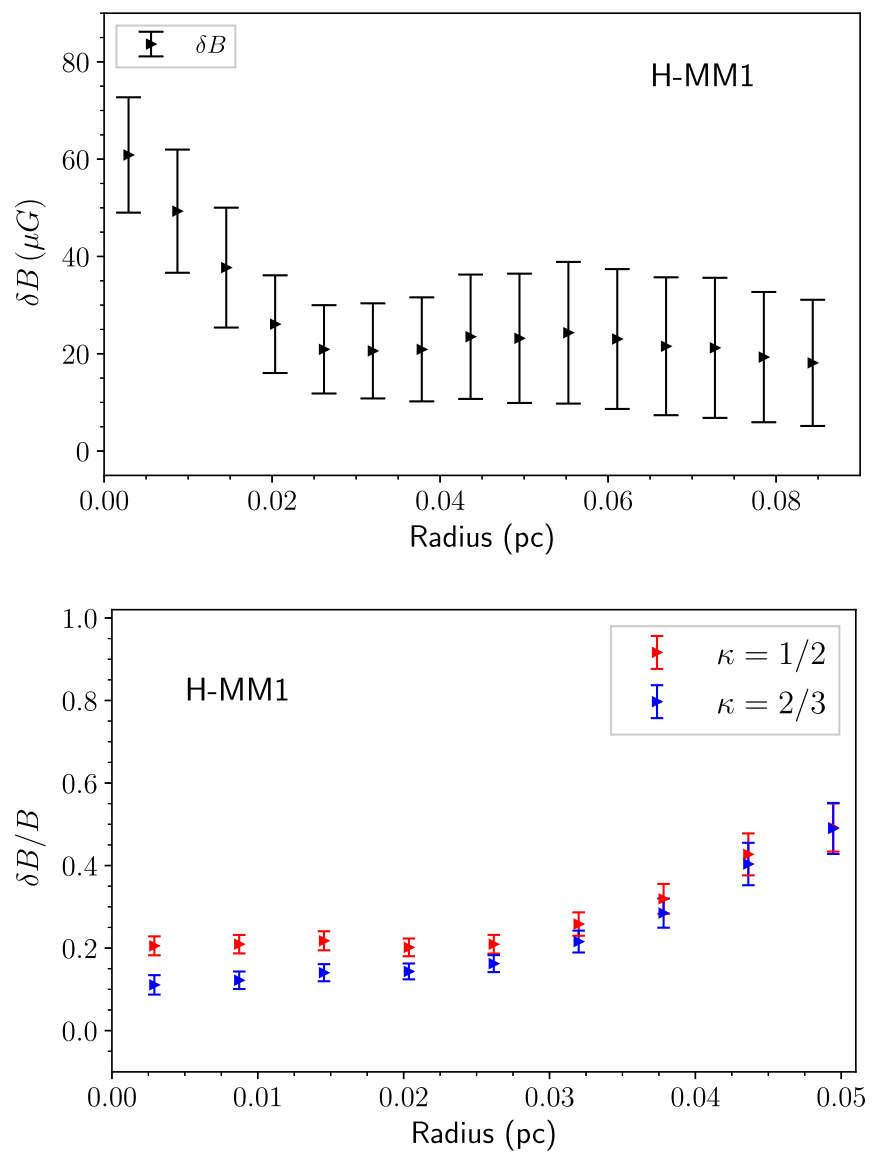

Figure 7. Top: the magnetic field fluctuation $\delta B$ in H-MM1 vs. radius. Bottom: the variation of $\delta B / B$ for $\kappa=1 / 2$ (red) and $\kappa=2 / 3$ (blue), respectively (assuming $\beta_{1}=0.5$ ). The radial profile for $\delta B / B$ is only within the transonic radius since the model (Equation (4)) is applied only in that region. The error bars in both cases are obtained using standard propagation of $1 \sigma$ error and Monte Carlo analysis.

However, for a near-flux-frozen condition, the field lines are pinched toward the central region of the dense core and resemble an hourglass morphology (Girart et al. 2006; Stephens et al. 2013). We rewrite the density profile of Equation (1) in normalized cylindrical coordinates $\xi \equiv x / r_{0}$ (we use $x$ as the radial coordinate) and $\zeta=z / r_{0}$ so that

$$
\frac{\rho(\xi, \zeta)}{\rho_{\mathrm{c}}}=\frac{\rho_{0} / \rho_{\mathrm{c}}}{\left[1+\xi^{2}+\zeta^{2}\right]^{p / 2}} .
$$

Using Equation (4) we can estimate the flux function

$$
\Phi(\xi, \zeta)=2 \pi r_{0}^{2} B\left(r_{\mathrm{c}}\right) \int_{0}^{\xi}\left[\frac{\rho_{0} / \rho_{\mathrm{c}}}{\left[1+\xi^{\prime 2}+\zeta^{2}\right]^{p / 2}}\right]^{\kappa} \xi^{\prime} d \xi^{\prime} .
$$

Here, we make the approximation that at each height the flux can be estimated from the scalar magnetic field strength obtained from Equation (4) rather than the local vertical component of $B$. This is equivalent to assuming that the field lines are not highly pinched in the observed region of the prestellar cores that are modeled here. An analytic solution to the above equation is only possible for the case where $p / 2=1$. We solve the above integral numerically and draw contours of constant magnetic flux. We note that each field line is a contour of constant enclosed flux (see Mouschovias 1976b). To estimate the enclosed mass through each of the flux tubes within the core, we integrate numerically. Figure 9 shows the mass-to-flux ratio of $\mathrm{H}-\mathrm{MM} 1$ as a function of radial distance from the center. As evident for both $\kappa=1 / 2$ and $2 / 3$, the mass-to-flux ratio $(\mu)$ is supercritical at the center and declines toward the core edge. However, the mass-to-flux ratio depends on the choice of $\beta$. See Section 4 for further discussion. Figure 9 shows that for a greater value of $\beta$ the mass-to-flux estimate increases and the entire core is supercritical. Figure 10 shows the profile of the mass-to-flux ratio for the six other cores studied in this paper. Most cores (namely H-MM2, Oph-E, Oph-FMM2b, and Oph-CN) show a similar decline of the mass-to-flux ratio toward the transonic radius. Oph-C is supercritical all the way to the core boundary for both the $\beta$ values. Oph-FE is close to the critical limit.

As an example of the magnetic field lines, we demonstrate the case of H-MM1, where we plot in Figure 11 the flux contours for the power-law model with index $\kappa=2 / 3$. To represent the field lines, we introduce a background field strength $\left(B_{\mathrm{u}}\right)$ and background density $\rho_{\mathrm{u}}$. The flux $\Phi$ is estimated using the Equation (9) but with the modified density expression normalized to background density $\rho_{\mathrm{u}}$ :

$$
\frac{\rho(r)}{\rho_{\mathrm{u}}}=1+\frac{\rho_{0} / \rho_{\mathrm{u}}}{\left[1+\xi^{2}+\zeta^{2}\right]^{p / 2}} .
$$

Here, the background density $\rho_{\mathrm{u}}$ is added to the core density $\rho(r)$. The flux lines in Figure 11 are normalized to $\Phi_{0}=2 \pi r_{0}^{2} B_{\mathrm{u}}$ for value $\rho_{0} / \rho_{\mathrm{u}}=300$. It should be noted that the mass-to-flux estimates are not strongly dependent on the background values, which are far less than the density in the vicinity of the transonic radius.

\section{Discussion}

We have introduced the CFS model, a new technique to predict the magnetic field strength profile of a dense core. This model is built on a similar premise as the DCF method, where the nonthermal velocity fluctuations are assumed to be Alfvénic. The use of $\delta B / B=\sigma_{\mathrm{NT}} / v_{\mathrm{A}}$ is common to both methods. In the CFS model, we measure $\delta B=\sigma_{\mathrm{NT}}(4 \pi \rho)^{1 / 2}$, unlike the DCF method that estimates $\delta B / B$ using the dispersion $(\delta \theta)$ in direction of the polarization vectors. Although similar to the DCF technique, the CFS model has a major advantage in that it predicts a field strength profile. The DCF model for a core gives only a core-average field strength estimate based on average density, average velocity dispersion, and average polarization angle dispersion. For well resolved core maps in the $\mathrm{NH}_{3}$ lines, the CFS model gives a finer scale prediction of field structure in a core based on our choice of $\delta B / B$ at the transonic radius. However, the CFS model does not model the transition zone where there is a sharp drop of the nonthermal line width.

The decease of the line width can be a consequence of damping of the Alfvén waves due to reflection or dissipation across a density gradient (Pinto et al. 2012). It could be also due to the drop in the ionization fraction at the transonic radius, leading to ambipolar diffusion damping of Alfvén waves. Thus, it is possible that non-ideal MHD effects may become relevant within the core. 

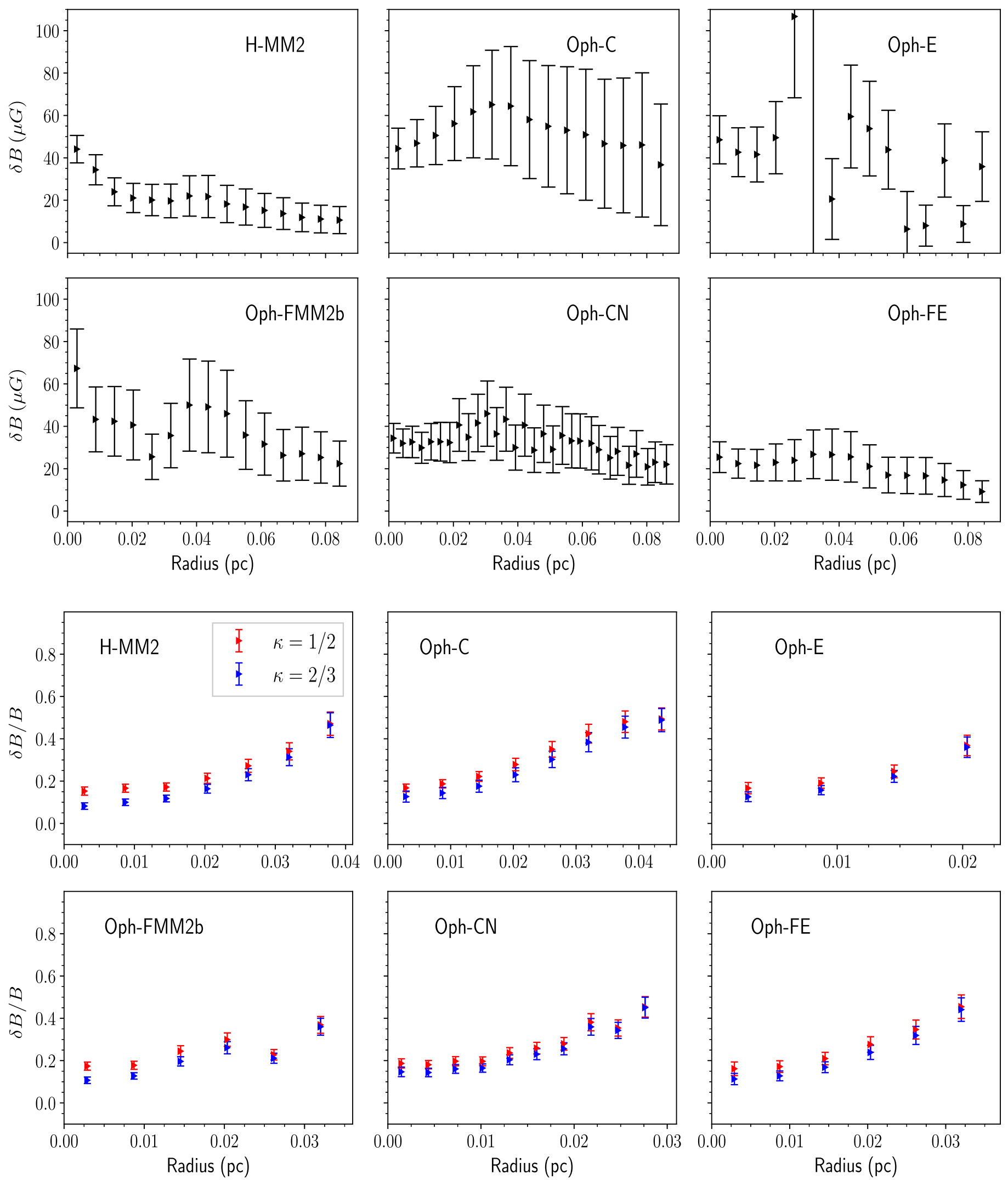

Figure 8. Top two panels: the variation of $\delta B$ across the six cores in Ophiuchus. Bottom two panels: the red and the blue lines show the variation of $\delta B / B$ for $\kappa=1 / 2$ and $\kappa=2 / 3$, respectively. The error bars in both the cases are obtained using a standard propagation of $1 \sigma$ error and Monte Carlo analysis.

In Appendix C, we consider the effect of ambipolar diffusion on wave propagation within the core. Equation (23) gives a modified version of the Alfvénic theory, which incorporates the correction term due to ambipolar diffusion. For conditions appropriate to a dense core, Equation (27) shows that the use of the flux-freezing relation, Equation (5), is approximately valid 


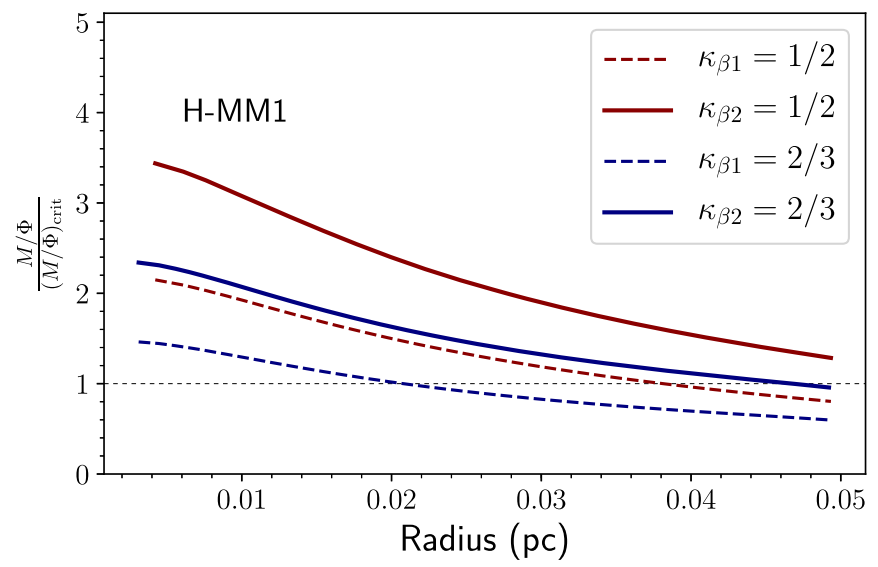

Figure 9. The normalized mass-to-flux ratio $\mu \equiv M / \Phi /(M / \Phi)_{\text {crit }}$ of H-MM1 as a function of radial distance from the center. The dashed and the solid lines are for $\beta_{1}=0.5$ and $\beta_{2}=0.8$, respectively. The core is mostly supercritical with $\mu>1$ (depending on the value of $\beta$ ) and is decreasing outward. The dotted horizontal line indicates the critical mass-to-flux ratio.

within the core. Furthermore, even though the Alfvén waves are damped within the core, their wavelengths are long enough that they can propagate above cutoff and can be responsible for the observed nonthermal line widths.

A significant source of uncertainty in the CFS model is the value of $\beta$. As seen previously, the magnetic field strength varies by $\approx 38 \%$ when the value of $\beta$ changes from 0.5 to 0.8 . Although results from turbulent simulations (for example Kudoh \& Basu 2003) do constrain the value of $\beta$ to be $<1$, there is still an allowed spread in the choice of $\beta$. Another possible approach is to assume a critical mass-to-flux ratio within the transonic radius and then derive a value of $\beta$ at the transonic radius. This yields a $\beta$ value for all cores that is close to 0.6, with the distribution having a mean and standard deviation of 0.57 and 0.16 , respectively. Overall, the assumptions of $\beta \lesssim 1$ at the transonic radius and $\mu \gtrsim 1$ within the core are mutually consistent. Furthermore, the average interior value of $\beta \equiv \delta \theta=0.12$ Kandori et al. (2017) for the starless core Fest 1-457 lies within the range of estimated values of $\beta$ inside the transonic radius of H-MM1 (see Figure 7).

Another source of uncertainty is in the approximation of cores as spheres in which there is a power-law relation $B \propto \rho^{\kappa}$ for the magnetic field strength. The cores are most likely spheroids that have at least some flattening along the magnetic field direction. The relation $B \propto \rho^{\kappa}$ actually applies to average quantities in an object that contracts with flux freezing; $\kappa=2 / 3$ appropriate for spherical contraction (Mestel 1966) and $\kappa=1 / 2$ appropriate for contraction with flattening along the magnetic field direction (Mouschovias 1976a). The spherical model of Mestel (1966) has features that are not present in our simplified spherical model in which $B \propto \rho^{\kappa}$ at every interior point. The magnetic field strength in the hourglass pattern calculated by Mestel (1966) is not spherically symmetric and has slightly different profiles along the cylindrical $r$ - (hereafter $x$-) and $z$-directions.

We compared our Equation (4) results to the Mestel (1966) model along both principal axes for clouds with central-to-surface (transonic radius) density ratios of 30 and 300, and found a maximum factor of 2 discrepancy. The values of the Mestel (1966) model differ most from our model along the $x$-direction, where they can be up to a factor of 2 greater, but they differ less along the $z$-direction, where they are less than those of our model. The differences decrease as the central-to-surface density ratio increases (see also Myers et al. 2018). In the flattened magnetohydrostatic equilibrium models of Mouschovias (1976a), the magnetic field strength at the center of the cloud is about a factor of 2 less than our central value, for an equilibrium cloud with a critical mass-toflux ratio and central-to-surface density ratio of about 20 . This means that the effective value of $\kappa$ is slightly less than $1 / 2$ at the center of that model. Figure 7 of Mouschovias (1976a) shows that the central value of $\kappa$ approaches $1 / 2$ as greater central density enhancements are obtained. This can also be seen in Figure 8 of Tomisaka et al. (1988).

In this paper, the nonthermal velocity dispersion derived from observed two-dimensional maps is used to approximate the nonthermal velocity dispersion $\sigma_{\mathrm{NT}}(r)$ as a function of spherical radius in three-dimensions. This approximation overestimates $\sigma_{\mathrm{NT}}(r)$ because it treats a map of the line-ofsight average as a function of map radius as though it were a map of the nonthermal velocity dispersion along a spherical radius. The line-of-sight column density is also used to derive a density that we take to be a function of spherical radius. By comparing numbers for a Plummer sphere with $p=2$, we find that the ratio of this line-of-sight average density at a map radius to the actual density at the same value of spherical radius is about 0.75 for a wide range of $r \geqslant 3 r_{0}$ from which most of the map information is obtained

With the CFS model, we have a new tool to study the spatial profile of magnetic fields in cores with high-resolution $\mathrm{NH}_{3}$ line maps. Both the magnetic field strength and the hourglass morphology can be predicted from our model. (See Myers et al. 2018 for a detailed model of hourglass morphology and comparison with a polarization map.) Furthermore, the CFS model provides a prediction of the radial profile of the polarization dispersion angle, if measurable. This opens up the possibility of using high spatial resolution polarimetry maps to test the idea of Alfvénic fluctuations in a way that is not possible with the DCF method alone. Some progress has recently been made in this direction by Kandori et al. (2018), who utilize the radial distribution of the polarization angle dispersion to estimate the magnetic field strength profile in the starless core FeSt 1-457.

\section{Conclusions}

The important results from the above study are summarized as follows:

1. All of the observed cores in the L1688 region of the Ophiuchus molecular cloud show a sharp decrease in their nonthermal line width as they become subthermal toward the center of the core. Furthermore, in the outer parts of H-MM1, H-MM2, Oph-C, and Oph-E, there is a substantial increase of $\sigma_{\mathrm{NT}}$ compared to $c_{\mathrm{s}}$.

2. The CFS model predicts $B(r)$, the magnetic field strength as a function of radius, which we estimate to be accurate within a factor $\sim 2$. It incorporates spatially resolved observations of the nonthermal velocity dispersion $\sigma_{\mathrm{NT}}$ and the gas density in a relatively circular dense core.

3. The CFS model yields an estimate of the profile of the magnetic field fluctuations $\delta B$ and the relative field fluctuation $\delta B / B$ inside the core.

4. We find that the condition $\delta B / B \lesssim 1$ at the edge of the core (where $\sigma_{\mathrm{NT}}=c_{\mathrm{s}}$ ) is consistent with a normalized mass-to-flux ratio $\mu \gtrsim 1$ inside the core. 

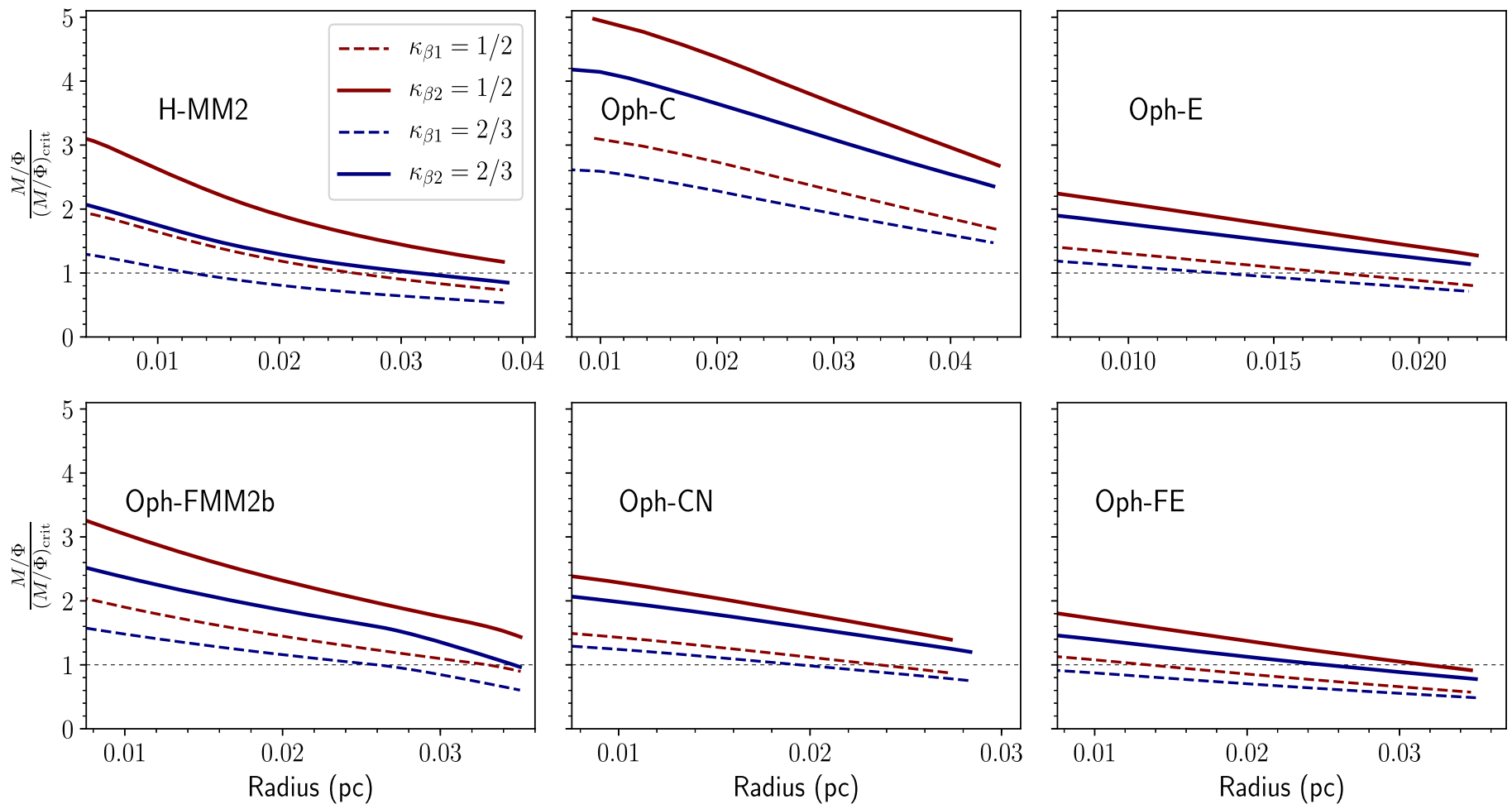

Figure 10. The normalized mass-to-flux ratio $\mu \equiv M / \Phi /(M / \Phi)_{\text {crit }}$ as a function of radial distance from the center for the remaining six cores in our sample. The dashed and the solid lines are for $\beta_{1}=0.5$ and $\beta_{2}=0.8$, respectively. The core is mostly supercritical with $\mu>1$ (depending on the value of $\beta$ ) and is decreasing outward. The dotted horizontal line indicates the critical mass-to-flux ratio.

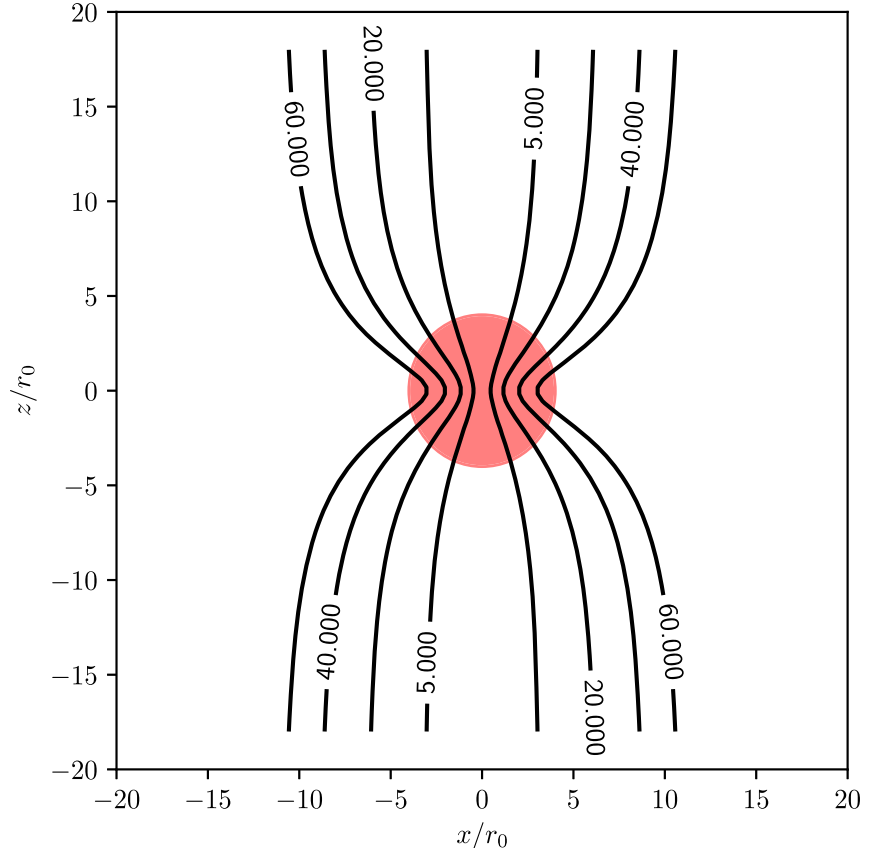

Figure 11. An illustration of the magnetic flux contours in H-MM1 for the power-law model with $\kappa=2 / 3$. The core parameters for H-MM1 $\left(n_{0}, r_{0}, r_{\mathrm{c}}\right)$ are taken from Table 1 . The peak density $\rho_{\mathrm{c}}$ is chosen to be 300 times the background. The marked flux lines are normalized to a background value $\Phi_{0}=2 \pi r_{0}^{2} B_{\mathrm{u}}$ (refer to the text for details). The $x$ - and $z$-axes are in units of $r_{0}=0.012 \mathrm{pc}$. The circle at the center represents the H-MM1 core of radius $r_{\mathrm{c}}=0.05 \mathrm{pc}$.
5. We map the mass-to-flux ratio of cores in Ophiuchus using the CFS model. The mass-to-flux ratio is decreasing radially outward from the center of the core.

We thank Sarah Sadavoy, Ian Stephens, Riwaj Pokhrel, Mike Dunham, and Tyler Bourke for fruitful discussions. We also thank the anonymous referee for comments that improved the presentation of results in this paper. S.B. is supported by a Discovery Grant from NSERC. J.E.P. acknowledges the financial support of the European Research Council (ERC; project PALs 320620). The National Radio Astronomy Observatory is a facility of the National Science Foundation operated under cooperative agreement by Associated Universities, Inc.

\section{Appendix A Annularly Averaged Spectra}

Figure 12 shows a $(25 \times 25)$ grid of $\mathrm{NH}_{3}(1,1)$ spectra around HMM-1. The center of the plot corresponds to the center of the H-MM1 core. For each concentric ring, we calculate the average spectra after aligning them in velocity using the LSR velocity maps. The averaged spectra for each of the rings are shown in the right panel. We apply the same procedure to obtain the averaged $\mathrm{NH}_{3}(1,1)$ and $\mathrm{NH}_{3}(2,2)$ spectra for all of the cores studied in this paper. These annularly averaged spectra are then used to extract the thermal and nonthermal components of the velocity dispersion, as described in Section 2.1. 

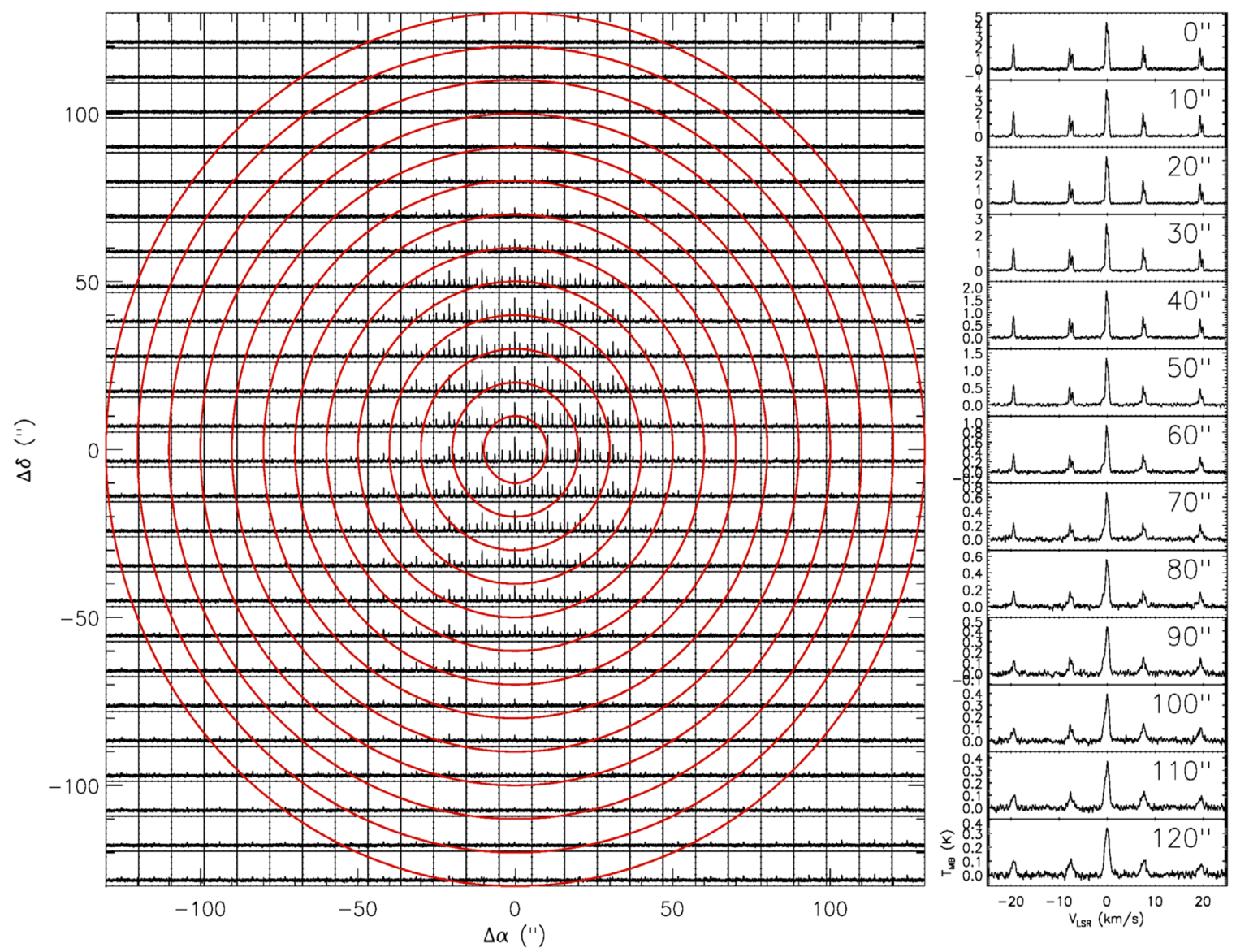

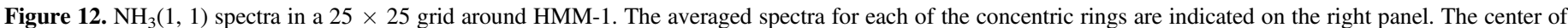

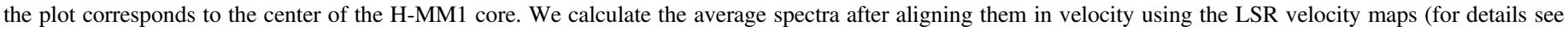
Section 2.1). The intensity of the averaged spectra decreases away from the core center.

\section{Appendix B \\ Mass and Flux of a Cylindrical Tube}

Here, we consider a simple case where the magnetic field lines are assumed to be vertically threading a spherical core in the plane of the sky. We calculate the enclosed mass within cylindrical tubes of constant magnetic field strength. We integrate the volume density $\rho(r)$ given in Equation (1) with $p / 2=1$ along the magnetic field lines (assumed to be vertical). (See Figure 13 for a schematic of the integration.) The column density is

$$
\begin{aligned}
\Sigma(x) & =2 \int_{0}^{\sqrt{r_{\mathrm{c}}^{2}-x^{2}}} \rho(s) d s \\
& =2 \int_{x}^{r_{\mathrm{c}}} \frac{\rho(r) r d r}{\sqrt{r^{2}-x^{2}}},
\end{aligned}
$$

where $x$ is the offset from the center in the midplane (see Dapp \& Basu 2009 for an analogous calculation). The column density is then

$$
\Sigma(x)=\frac{2 r_{0}^{2} \rho_{0}}{\sqrt{\left(x^{2}+r_{0}^{2}\right)}} \arctan \left(\frac{\sqrt{r_{\mathrm{c}}-x^{2}}}{\sqrt{r_{0}+x^{2}}}\right) .
$$

We find the mass of the cylindrical tubes by integrating the column density from the center to a given distance $x$ in the midplane:

$$
M(x)=2 \pi \int_{0}^{x} x^{\prime} \Sigma\left(x^{\prime}\right) d x^{\prime}
$$

Inserting Equation (12) in Equation (13) and integrating, we find

$$
\begin{aligned}
M(x)= & 4 \pi \rho_{0} r_{0}^{2}\left[r_{\mathrm{c}}-r_{0} \arctan \frac{r_{\mathrm{c}}}{r_{0}}-\sqrt{r_{\mathrm{c}}^{2}-x^{2}}\right. \\
& \left.+\sqrt{r_{0}^{2}-x^{2}} \arctan \left(\frac{\sqrt{r_{\mathrm{c}}-x^{2}}}{\sqrt{r_{0}+x^{2}}}\right)\right] .
\end{aligned}
$$

The corresponding magnetic flux is estimated by integrating the magnetic field profile in the horizontal midplane of the core:

$$
\Phi(x)=2 \pi \int_{0}^{x} B x^{\prime} d x^{\prime} .
$$

Using Equations (4) and (1) in the above equation yields

$$
\begin{aligned}
\Phi(x)= & 2 \pi B_{\mathrm{c}} r_{0}^{2}\left(\frac{n_{0}}{n_{\mathrm{c}}}\right)^{\kappa}\left[\frac{\left(1+\left(x / r_{0}\right)^{2}\right)^{(1-\kappa)}}{2-2 \kappa}\right. \\
& \left.-\frac{1}{2-2 \kappa}\right] .
\end{aligned}
$$

For the more general case where $p / 2 \neq 1$, we can use numerical integration to estimate the mass and the flux of a given core. 


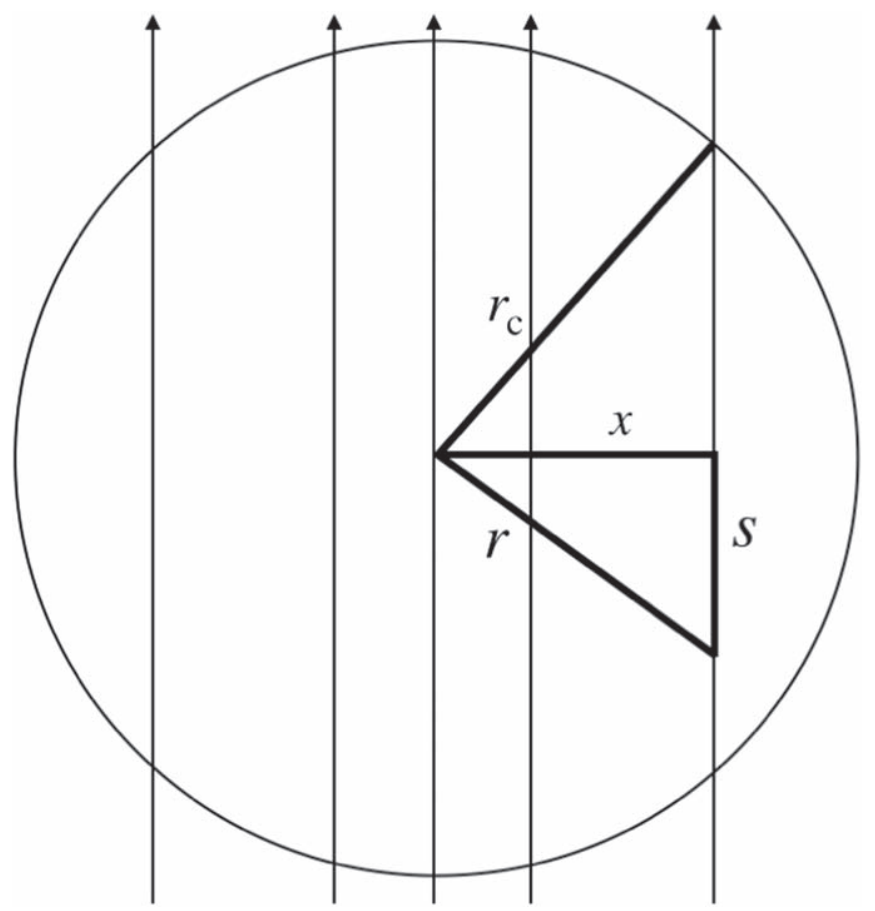

Figure 13. Schematic illustration of a cut through a spherical core of radius $r_{\mathrm{c}}$. The vertical arrows represent the magnetic field lines in the plane of the sky. The column density $\Sigma$ as a function of the offset $x$ is obtained by integrating along the direction $s$ parallel to the magnetic field lines.

\section{Appendix C Dispersion Relation}

The dispersion relation of Alfvén waves in a partially ionized medium (Pinto et al. 2012) in the long wavelength limit (i.e., $\left.\lambda \gg 2 \pi v_{\mathrm{A}} \tau_{\mathrm{ni}}\right)$ is

$$
\omega^{2}-k^{2} v_{\mathrm{A}}^{2}+i \eta_{\mathrm{AD}} k^{2} \omega=0
$$

Here, $\eta_{\mathrm{AD}}=v_{\mathrm{A}}^{2} \tau_{\mathrm{ni}}$ is the ambipolar diffusion resistivity and $\tau_{\mathrm{ni}}=\left(\gamma_{\mathrm{ni}} \rho_{\mathrm{i}}\right)^{-1}$ is the mean neutral-ion collision time in terms of the drag coefficient

$$
\gamma_{\mathrm{ni}}=\frac{\langle\sigma w\rangle_{\mathrm{in}}}{1.4\left(m_{\mathrm{n}}+m_{\mathrm{i}}\right)}
$$

(Basu \& Mouschovias 1994), and the ion density $\rho_{\mathrm{i}}$. In the above equation, $\langle\sigma w\rangle_{\text {in }}$ is the average collision rate between the ions of mass $m_{\mathrm{i}}$ and neutrals of mass $m_{\mathrm{n}}$. On rearranging, Equation (17) is rewritten as

$$
k^{2}=\frac{\omega^{2}}{v_{\mathrm{A}}^{2}}\left[\frac{1}{1+i \omega \tau_{\mathrm{ni}}}\right]
$$

In the limit $\omega \tau_{\mathrm{ni}} \ll 1$, Equation (19) on binomial expansion yields

$$
k=\frac{\omega}{v_{\mathrm{A}}}\left(1-i \frac{1}{2} \omega \tau_{\mathrm{ni}}\right) .
$$

Defining $\xi=(1 / 2) \omega \tau_{\text {ni }}$ as a dimensionless parameter, we can represent Equation (20) in terms of a magnitude and a phase $\theta$ :

$$
\left|\frac{k}{\omega}\right|=\frac{1}{v_{\mathrm{A}}} \sqrt{1+\xi^{2}}\left|e^{i \theta}\right|
$$

Using Equation (20) to replace $k$ in Equation (17) from Pinto et al. (2012), we derive the relation between the amplitude of fluctuation of the neutral velocity $u_{\mathrm{n} 0}$ to the fluctuation of the magnetic field $\delta B$. In the long wavelength limit, we get

$$
\left|u_{\mathrm{n} 0}\right|=v_{\mathrm{A}} \frac{|\delta B|}{B} \sqrt{1+\xi^{2}}
$$

If $\xi \ll 1$, then

$$
\left|u_{\mathrm{n} 0}\right| \simeq v_{\mathrm{A}} \frac{|\delta B|}{B}\left[1+\frac{1}{2} \xi^{2}\right] .
$$

This gives a modified version for the Alfvénic theory, which incorporates the correction term due to ambipolar diffusion. Equation (23) is equivalent to Equation (5), if we equate $\sigma_{\mathrm{NT}}=\left|u_{\mathrm{n} 0}\right|$ and take the limit $\xi \rightarrow 0$. Again assuming that $\xi \ll 1$ (which we will later verify), we apply the standard dispersion relation of ideal Alfvén waves, $\omega=v_{\mathrm{A}} k$, and express $\xi$ in terms of wavenumber $k$,

$$
\xi^{2}=\left(\frac{v_{\mathrm{A}} \tau_{\mathrm{ni}} k}{2}\right)^{2} .
$$

Using Equation (18) to replace $\tau_{\mathrm{ni}}$ in terms of the drag coefficient $\gamma_{\text {ni }}$ and ion density, we get

$$
\xi^{2}=\frac{1}{4} \frac{B^{2}}{4 \pi \rho_{\mathrm{n}}} k^{2}\left[1.4 \frac{m_{\mathrm{i}}+m_{\mathrm{n}}}{\rho_{\mathrm{i}}\langle\sigma w\rangle_{\mathrm{in}}}\right]^{2} .
$$

We can estimate the above quantities in Equation (24) by specifying appropriate values relevant for dense cores embedded in molecular clouds. For example, if $B \simeq 30 \mu G$ and $\rho_{\mathrm{n}}=m_{\mathrm{n}} n_{0}$, where $n_{0}=10^{4} \mathrm{~cm}^{-3}$ is the number density of neutrals and $m_{\mathrm{n}}=2.33 \times m_{\mathrm{H}}$, the Alfvén speed $v_{\mathrm{A}}=0.4 \mathrm{~km} \mathrm{~s}^{-1}$. Furthermore, for $\langle\sigma w\rangle_{\mathrm{in}}=1.69 \times 10^{-9} \mathrm{~cm}^{-3} \mathrm{~s}^{-1}$ and $m_{\mathrm{i}}=29 \times m_{\mathrm{H}}$, the drag coefficient $\gamma_{\mathrm{ni}}=2.3 \times 10^{13} \mathrm{~cm}^{3} \mathrm{~g}^{-1} \mathrm{~s}^{-1}$. The ion density $\rho_{\mathrm{i}}$ is determined by the approximate relation

$$
\rho_{\mathrm{i}}=m_{\mathrm{i}} K n_{0}^{1 / 2}=1.45 \times 10^{-23} \mathrm{~g} \mathrm{~cm}^{-3},
$$

where $K=3 \times 10^{-3} \mathrm{~cm}^{-3}$ (Elmegreen 1979). This gives $\tau_{\mathrm{ni}}=\left(\gamma_{\mathrm{ni}} \rho_{\mathrm{i}}\right)^{-1}=3 \times 10^{9} \mathrm{~s}$. The wavenumber $k=(2 \pi) / \lambda$ of interest will roughly correspond to a wavelength $\lambda \simeq 0.1 \mathrm{pc}$, i.e., about equal to the core diameter. This yields

$$
\xi=1.3 \times 10^{-3}\left(\frac{v_{\mathrm{A}}}{0.4 \mathrm{~km} \mathrm{~s}^{-1}}\right)\left(\frac{\tau_{\mathrm{ni}}}{3 \times 10^{9} \mathrm{~s}}\right)\left(\frac{0.1 \mathrm{pc}}{\lambda}\right),
$$

such that $\xi \ll 1$ (justifying the approximation we made in Equation (23)) in a dense core. This is equivalent to the waves having wavelength $\lambda \gg \lambda_{\mathrm{cr}}$, where $\lambda_{\mathrm{cr}}=\pi v_{\mathrm{A}} \tau_{\mathrm{ni}}$, (see Equation (15) in Pinto et al. 2012) is the critical wavelength for wave propagation, i.e., wavelengths shorter than $\lambda_{\mathrm{cr}}$ are critically damped. Thus, the Alfvén waves can still propagate within the core and be responsible for the observed nonthermal line widths. The condition $\xi \ll 1$ continues to apply for wavelengths $\lambda$ significantly smaller that $0.1 \mathrm{pc}$, as can be seen from Equation (27).

\section{ORCID iDs}

Sayantan Auddy (ib https://orcid.org/0000-0003-3784-8913 Philip C. Myers (iD https://orcid.org/0000-0002-2885-1806 Shantanu Basu (iD https://orcid.org/0000-0003-0855-350X 
Jorma Harju (1) https://orcid.org/0000-0002-1189-9790

Jaime E. Pineda (1) https://orcid.org/0000-0002-3972-1978

Rachel K. Friesen (1) https://orcid.org/0000-0001-7594-8128

\section{References}

Andersson, B.-G., Lazarian, A., \& Vaillancourt, J. E. 2015, ARA\&A, 53, 501 André, P., Basu, S., \& Inutsuka, S. 2009, in The Formation and Evolution of Prestellar Cores, ed. G. Chabrier (Cambridge: Cambridge Univ. Press), 254 Arzoumanian, D., André, P., Didelon, P., et al. 2011, A\&A, 529, L6 Auddy, S., Basu, S., \& Kudoh, T. 2018, MNRAS, 474, 400

Basu, S., Ciolek, G. E., Dapp, W. B., \& Wurster, J. 2009a, NewA, 14, 483

Basu, S., Ciolek, G. E., \& Wurster, J. 2009b, NewA, 14, 221

Basu, S., \& Mouschovias, T. C. 1994, ApJ, 432, 720

Benson, P. J., \& Myers, P. C. 1989, ApJS, 71, 89

Caselli, P., Benson, P. J., Myers, P. C., \& Tafalla, M. 2002, ApJ, 572, 238

Chandrasekhar, S., \& Fermi, E. 1953, ApJ, 118, 113

Chen, C.-Y., \& Ostriker, E. C. 2014, ApJ, 785, 69

Crutcher, R. M. 1999, ApJ, 520, 706

Crutcher, R. M. 2012, ARA\&A, 50, 29

Danby, G., Flower, D. R., Valiron, P., Schilke, P., \& Walmsley, C. M. 1988, MNRAS, 235, 229

Dapp, W. B., \& Basu, S. 2009, MNRAS, 395, 1092

Davis, L., Jr., \& Greenstein, J. L. 1951, ApJ, 114, 206

Elmegreen, B. G. 1979, ApJ, 232, 729

Fiedler, R. A., \& Mouschovias, T. C. 1993, ApJ, 415, 680

Friesen, R. K., Pineda, J. E., Rosolowsky, E., et al. 2017, ApJ, 843, 63

Gammie, C. F., Lin, Y.-T., Stone, J. M., \& Ostriker, E. C. 2003, ApJ, 592, 203

Girart, J. M., Rao, R., \& Marrone, D. P. 2006, Sci, 313, 812

Goodman, A. A., Barranco, J. A., Wilner, D. J., \& Heyer, M. H. 1998, ApJ, 504,223

Ho, P. T. P., \& Townes, C. H. 1983, ARA\&A, 21, 239

Hoang, T., \& Lazarian, A. 2008, MNRAS, 388, 117

Jeans, J. H. 1929, The Universe Around Us (Cambridge: Cambridge Univ. Press)

Jijina, J., Myers, P. C., \& Adams, F. C. 1999, ApJS, 125, 161

Jørgensen, J. K., Johnstone, D., Kirk, H., et al. 2008, ApJ, 683, 822

Kandori, R., Tamura, M., Tomisaka, K., et al. 2017, ApJ, 848, 110

Kandori, R., Tomisaka, K., Tamura, M., et al. 2018, ApJ, 865, 121

Kirk, J. M., Ward-Thompson, D., \& André, P. 2005, MNRAS, 360, 1506

Klessen, R. S. 2001, ApJ, 556, 837

Kudoh, T., \& Basu, S. 2003, ApJ, 595, 842

Kudoh, T., \& Basu, S. 2011, ApJ, 728, 123

Kudoh, T., \& Basu, S. 2014, ApJ, 794, 127
Lada, C. J., Alves, J. F., \& Lombardi, M. 2007, in Protostars and Planets V, ed. B. Reipurth, D. Jewitt, \& K. Keil (Tucson, AZ: Univ. Arizona Press), 3

Lada, E. A., Strom, K. M., \& Myers, P. C. 1993, in Protostars and Planets III, ed. E. H. Levy \& J. I. Lunine (Tucson, AZ: Univ. Arizona Press), 245

Larson, R. B. 1985, MNRAS, 214, 379

Larson, R. B. 2003, RPPh, 66, 1651

Lazarian, A., \& Hoang, T. 2007, MNRAS, 378, 910

Loren, R. B. 1989, ApJ, 338, 902

Machaieie, D. A., Vilas-Boas, J. W., Wuensche, C. A., et al. 2017, ApJ, 836, 19

Marsh, K. A., Kirk, J. M., André, P., et al. 2016, MNRAS, 459, 342

Masters, J., Garwood, B., Langston, G., \& Shelton, A. 2011, in ASP Conf. Ser. 442, Astronomical Data Analysis Software and Systems XX, ed. I. N. Evans et al. (San Francisco, CA: ASP), 127

Mestel, L. 1966, MNRAS, 133, 265

Mestel, L., \& Spitzer, L., Jr. 1956, MNRAS, 116, 503

Mestel, L., \& Strittmatter, P. A. 1967, MNRAS, 137, 95

Mouschovias, T. C. 1976a, ApJ, 207, 141

Mouschovias, T. C. $1976 \mathrm{~b}$, ApJ, 206, 753

Mouschovias, T. C. $1979, \mathrm{ApJ}, 228,475$

Myers, P. C. 1983, ApJ, 270, 105

Myers, P. C., Basu, S., \& Auddy, S. 2018, ApJ, 868, 51

Myers, P. C., \& Benson, P. J. 1983, ApJ, 266, 309

Nakamura, F., \& Li, Z.-Y. 2005, ApJ, 631, 411

Nakano, T., \& Nakamura, T. 1978, PASJ, 30, 671

Padoan, P., Nordlund, A., \& Jones, B. J. T. 1997, MNRAS, 288, 145

Pattle, K., Ward-Thompson, D., Kirk, J. M., et al. 2015, MNRAS, 450, 1094

Pineda, J. E., Goodman, A. A., Arce, H. G., et al. 2010, ApJL, 712, L116

Pinto, C., Verdini, A., Galli, D., \& Velli, M. 2012, A\&A, 544, A66

Rydbeck, O. E. H., Sume, A., Hjalmarson, A., et al. 1977, ApJL, 215, L35

Shu, F. H., Adams, F. C., \& Lizano, S. 1987, ARA\&A, 25, 23

Stephens, I. W., Looney, L. W., Kwon, W., et al. 2013, ApJL, 769, L15

Teixeira, P. S., Lada, C. J., \& Alves, J. F. 2005, ApJ, 629, 276

Tomisaka, K., Ikeuchi, S., \& Nakamura, T. 1988, ApJ, 335, 239

Walmsley, C. M., \& Ungerechts, H. 1983, A\&A, 122, 164

Ward-Thompson, D., Scott, P. F., Hills, R. E., \& Andre, P. 1994, MNRAS, 268, 276

Wilking, B. A., Gagné, M., \& Allen, L. E. 2008, in Handbook of Star Forming Regions, Volume II: The Southern Sky ASP Monograph Publications, Vol. 5, ed. B. Reipurth (San Francisco, CA: ASP), 351

Wilking, B. A., \& Lada, C. J. 1983, ApJ, 274, 698

Williams, J. P., Blitz, L., \& McKee, C. F. 2000, in Protostars and Planets IV, ed. V. Mannings, A. P. Boss, \& S. S. Russell (Tucson, AZ: Univ. Arizona Press), 97

Wolleben, M., \& Reich, W. 2004, A\&A, 427, 537 